\section{A FUNCTIONAL GENETIC SCREEN UNCOVERS REGULATORS OF INTRATUMORAL MACROPHAGE FUNCTION AND REVEALS CD24 AS A NOVEL TARGET FOR CANCER IMMUNOTHERAPY BY MACROPHAGES}

${ }^{1}$ Amira Barkal*, ${ }^{2}$ Rachel Brewer, ${ }^{2}$ Irving Weissman. 'Brigham and Women's Hospital, Boston, MA, USA; ${ }^{2}$ Stanford University School of Medicine, Stanford, CA, USA

Background Cancer cells are capable of evading clearance by macrophages through the overexpression of anti-phagocytic, innate immune checkpoint molecules called 'don't eat me' signals, including CD47, ${ }^{1}$ PD-L1, ${ }^{2}$ and MHC class I. ${ }^{3}$ Monoclonal antibodies that antagonize the interaction of 'don't eat me' signals with their macrophage-expressed receptors have demonstrated therapeutic potential in several cancers. However, variability in the magnitude and durability of the responses to these agents has suggested the presence of additional, as yet unknown innate immune checkpoints. Here, we present a functional screening platform which identifies tumor-specific regulators of intratumoral macrophage function. We show that CD24 is a dominant innate immune checkpoint in many solid tumors, including ovarian cancer and breast cancer. ${ }^{4}$

Methods By applying our screening method, we uncovered the novel innate immune checkpoint molecule, CD24. To characterize the role of $\mathrm{CD} 24$ as a macrophage checkpoint, we leveraged the MCF-7 human xenograft tumor model and the ID8 syngeneic ovarian cancer tumor model. We evaluated the anti-tumor effect of CD24 antagonism through genetic ablation experiments in addition to therapeutic CD24 monoclonal antibody $(\mathrm{mAb})$ blockade. We also utilized primary human immune cells and tumor specimens to assess the effect of CD24 blockade either alone or in combination with additional tumor-targeting antibodies.

Results We demonstrate that CD24 promotes immune evasion through its interaction with the inhibitory macrophage receptor Siglec-10. Genetic ablation of either CD24 or Siglec-10, as well as blockade of the CD24-Siglec-10 interaction using monoclonal antibodies, robustly augmented the phagocytosis of all CD24-expressing human tumors that we tested. Therapeutic blockade of CD24 resulted in a macrophage-dependent reduction of tumor growth in vivo and an increase in survival time. The therapeutic efficacy of anti-CD24 mAbs was enhanced when combined with a second anti-tumor antibody. In particular, dual treatment of HER2-positive breast cancers with anti-CD24 mAb and trastuzumab, augmented phagocytosis relative to either treatment alone, even among cancers with inherent trastuzumab resistance (figure 1).

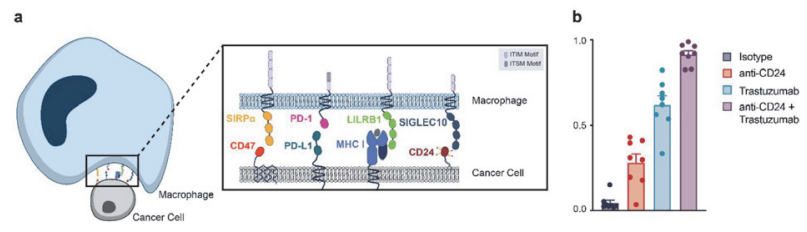

Abstract 261 Figure 1 Macrophage checkpoints are therapeutic targets. (A) There are four defined innate immune checkpoint signaling axes which exist between macrophages and cancer cells, which all rely on ITIM or ITSM signaling on the cytoplasmic side of the macrophage. (B) Phagocytosis of BT-474 ( $n=8$ donors) in the presence of anti-CD24 $\mathrm{mAb}$, anti-HER2 mAb or dual treatment, compared with IgG control.
Conclusions These data reveal CD24 as a highly expressed, anti-phagocytic signal in several cancers, and demonstrate the therapeutic potential for CD24 blockade in cancer immunotherapy, either alone or in combination with existing anticancer treatments. Collectively, this work suggests a new paradigm that innate immune checkpoints are redundant and employed in a tissue-specific and even tumor-specific manner, and makes clear the need to measure the collective expression of these 'don't eat me' signals in order to optimize patient responses to both innate and adaptive immunotherapies.

\section{REFERENCES}

1.. Majeti $R$, et al. CD47 is an adverse prognostic factor and therapeutic antibody target on human acute myeloid leukemia stem cells. Cell 2009;138: 286-299.

2.. Gordon SR, et al. PD-1 expression by tumour-associated macrophages inhibits phagocytosis and tumour immunity. Nature 2017:545:495-499.

3.. Barkal AA, et al. Engagement of MHC class I by the inhibitory receptor LILRB1 suppresses macrophages and is a target of cancer immunotherapy. Nat Immunol 2018:19:76-84

4.. Barkal AA, Brewer RE, Markovic M, Kowarsky MA, Barkal SA, Zaro BW, Krishnan V, Hatakeyama J, Dorigo O, Barkal LJ, Weissman IL. CD24 signaling through macrophage siglec-10 is a new target for cancer immunotherapy. Nature 2019;572:392-396.

Ethics Approval The Human Immune Monitoring Center Biobank and the Stanford Tissue Bank all received IRB approval from the Stanford University Administrative Panels on Human Subjects Research and complied with all ethical guidelines for human subjects research to obtain samples from patients with ovarian cancer and breast cancer, and received informed consent from all patients.

http://dx.doi.org/10.1136/jitc-2021-SITC2021.261 